\title{
Sum-of-Sinusoids-Based Fading Channel Models with Rician K-factor and Vehicle Speed Ratio in Vehicular Ad Hoc Networks
}

\author{
Yuhao Wang and Xing Xing \\ School of Information Engineering, Nanchang University
}

China

\section{Introduction}

In this chapter, we propose an extended reference model and two novel sum-of-sinusoids (SoS) models (statistical and deterministic simulation models) propagation models considering the Rician K-factor and vehicle speed ratio in Vehicular Ad Hoc Networks (VANETs). Our models consider comprehensive scene of wave propagation, including I2V (infrastructure-to-vehicle) channels with a LOS or NLOS environment, IVC (inter-vehicle communication) channels with a LOS or NLOS environment. The analysis of the statistical properties of the proposed models show that the statistics of the new models match those of the reference model at a large range of normalized time delays. The proposed models show improved approximations to the desired auto-correlation and faster convergence with the increase of Rician K-factor and vehicle speed ratio.

\section{Background}

VANETs have been envisioned for safety, traffic management, and commercial applications, such as information notices of hazardous road conditions, traffic congestion, or sudden stops, furthermore, commercial services (e.g., data exchange, infotainment, rear-seat multiplayer games) (Boban et al., 2009)-(Abbas et al., 2010). Recently, the Quality of Service (QoS) and network security are used to determine the feasibility of such applications. To achieve the desirable QoS and network security, many techniques focusing on the design and optimization of routing protocol in VANETs have been proposed in (Lwinmuller et al., 2006)-(Saleet et al., 2010). However, the fundamental issue centers on the accurate description of the characteristics and mechanism of wave propagation in VANETs, so it is essential to establish a reasonable radio propagation model for VANET channels, which help to understand the characteristics of the channel and take some effective steps to improve the QoS and network security.

Direct communication between vehicles in VANETs may be supported by the deployment of Mobile Ad Hoc Networks (MANETs), which does not rely on fixed infrastructure and can accommodate a constantly evolving network topology (Boban et al., 2009; Vahid, 2009). More recently, infrastructure-to-vehicle (I2V) and inter-vehicle communication (IVC) links are being evaluated for a variety of applications. I2V channels is similar to the traditional cellular systems, the base station is stationary, and only the mobile terminal are in motion. However, 
for IVC channels, a variety of applications, such as intelligent transportation systems and ad hoc networks, are based on mobile-to-mobile communications. Both the base station and mobile terminal are all in motion and the transmitted and received signals are all affected by the surrounded scatterers. So channel modeling in VANETs should be considered the both characteristics in I2V and IVC channels. More recently, infrastructure-to-vehicle (I2V) and inter-vehicle communication (IVC) links are being evaluated for VANETs and a LOS or NLOS environment should also be considered. The I2V and IVC channels can be distinguished by vehicle speed ratio and the difference of LOS and NLOS environment can also be represented by Rician $\mathrm{K}$ factor. Therefore, a comprehensive channel is needed to wholly describe the scene of wave propagation for VANETs.

An important factor of a vehicular channel model is the mobility (Gowrishankar et al., 2007; Yoon \& Noble, 2006) by including the mobility of nodes and the channel variability. Channel variability, is not well modeled in today's wireless vehicle networks. (Pawlikowski et al., 2002) reports that simplistic models may not be practical and it is also different to draw conclusions on the real performance of the upper layers. Designers require statistical models that can accurately capture the characteristic of propagation behavior observed at both mobile vehicles (Michelson \& Chuang, 2006).

Currently, free space and two ray ground channel models are the most popular propagation models for simulation in vehicular wireless networks (NS, 2000). For the free space channel model, it describes an ideal propagation characteristic, and the received power depends on the transmitted power, the gain of antenna, and the distance of transmitter-receiver. While for the two way ground model, it assumes that the received signal is the sum of the direct line of sight path and the reflected path from the ground. However, the model does not take obstacles into consideration. It is also too ideal for short transmitter-receiver separation distances, as it assumes that signals have a perfect 250m radius range. On the other hand, QualNet supports open-space propagation as well as stochastic propagation models such as Rayleigh, Rician and Log-normal fading, in which all models describe the time-correlation of the received signal power. Rayleigh model considers indirect paths between the transmitter and the receiver, while Rician model considers when there is one dominant path and multiple indirect signals. OPNet supports open-space propagation models as well as an enhanced open-space model that accounts for hills, foliage and atmospheric affects(OPNET, 2000). Furthermore, obstacle effects are combined in (Jardosh et al., 2006; Jradosh et al., 2005; Mahajan et al., 2007), but the propagation characteristic is limited to line-of-sight. (Stepanoy \& Rothermel, 2008) applies a radio planning tool and validates the evaluation for the impact of a more realistic propagation by a set of measurements.

In a dense urban area, path loss, shadow fading and short-term variants are the main factors affecting the communication quality. Path loss and shadowing fading determine the effective communication distance between two adjacent vehicles, while multi-path and Doppler spectrum caused by the sum of absolute speeds of individual nodes affect the quality of service (QoS) in inter-vehicle networks. However, it is noted that some of these effects can be avoided, such as by increasing the height of the antenna, and the inerratic variations is just relative to the distance between transmitter and receiver. Here, the model is focused on the short-term variants, especially for Doppler spectrum model caused by both high mobile vehicles. The Doppler spectrum model in (Clarke, 1968; Gans, 1972) for wireless cellular network cannot really be used for link between double mobile nodes. Akki and Haberp(Akki \& Haber, 1989) consider a Doppler spectrum model for radio link between 
double mobile nodes in a two-dimensional (2-D) uniform scattering environment. It is a deterministic channel model without considering the specific characteristics, such as the effect of antenna and dynamic distribution of received multi-path wave.

Motivated by the recently presented IVC fading channel models in (Patel et al., 2005; Zajic \& Stuber, 2006; Wang et al., 2009), we propose novel statistical and deterministic SoS models for Rician channels in VANETs. As described in (Patel et al., 2005), the properties, like auto-correlation and cross-correlation of the statistical models, differ for each simulation trial, but converge in a statistical sense to the desired properties over an infinite number of simulations. Therefore, such models are termed statistical models. In contrast, the properties of deterministic model, are identical for all simulation trials, hence, they can be predetermined, such models are called deterministic models. We provide detailed simulation results to verify and compare the performance of the proposed models in next sections.

The remainder of this chapter is organized as follows. Section 3 discusses the related work reported in the literature. In Section 4, we extend Akki and Haber's mathematical reference model for IVC channels by introducing the line-of-sight (LOS) components and derive the statistical properties of the extended model. Section 5 establishes new statistical and deterministic SoS simulation models. Their statistical properties are also derived and verified in this section. In Section 6, performance analysis is carried out through comparisons between the reference and the two SoS models. At last, the conclusion remarks are given in Section 7.

\section{Related research work}

A number of techniques have been proposed for the modeling and simulation of I2V channels. Among them, Clarke (Clarke, 1968) proposed the statistical theory of mobile-radio reception, and a power-spectral theory of propagation in the mobile-radio was developed by Gans in (Gans, 1972). The Jakes' simulator (Jakes, 1994; Dent et al., 1993), which is a simplified simulation model of Clarke's model (Clarke, 1968), has been widely used for frequency nonselective Rayleigh fading channels. Various modified models (Patzold et al., 1998)-(Li \& Huang, 2002) and improvements (Xiao \& Zheng, 2002)-(Zheng \& Xiao, 2003) of Jakes' simulator for generating multiple uncorrelated fading waveforms needed for modeling frequency selective fading channels and multiple-input multiple-output (MIMO) channels have been reported. It is commonly perceived that Jakes' simulator (and its modifications) is more computationally efficient than Clarke's model since Jakes' simulator needs only one fourth the number of low-frequency oscillators as needed in Clarke's model. However, recently Pop and Beaulieu (Pop \& Beaulieu, 2001) put forward a view that Jakes' simulator and its variants are not wide sense stationary (WSS), and that the reduction of simulator oscillators based on azimuthal symmetries lacks sufficient basis (Xiao et al., 2006). They improved the simulator by introducing random phase shifts in the low-frequency oscillators to remove the stationary problem in (Pop \& Beaulieu, 2001). But Xiao and Zheng (Zheng \& Xiao, 2003) gave a statistical analysis of Clarke's model with a finite number of sinusoids and showed that the Pop-Beaulieu simulator has also deficiencies in some of its higher-order statistics. it was further proved in (Xiao et al., 2002) that second-order statistics of the quadrature components and the envelope do not match the desired ones. Moreover, even in the limit as the number of sinusoids approaches infinity, the auto-correlations and cross-correlations of the quadrature components, and the auto-correlation of the squared envelope of the improved simulator, fail to match the desired correlations. Also, Jakes's 
original simulator and published modified versions, have similar problems with these second-order statistics. In (Xiao et al., 2006), Xiao and Zheng proposed a statistical SoS model for I2V channels which employs a zero-mean stochastic sinusoid as the specular LOS component, in contrast to previous Rician fading simulators that utilize a non-zero deterministic specular component. The statistical properties of the new simulators are confirmed by extensive simulation results, showing good agreement with theoretical analysis in all cases.

Channel modeling in VANETs should be considered the both characteristics in I2V and IVC channels. Those I2V channel models may not fully reflect the mobility characteristics of VANET channels. Several works in the open literature have been studied in this area (Akki \& Haber, 1989)-(Patel et al., 2003). The theoretical analysis of the IVC channels for urban and suburban land communication channels was first developed by Akki and Haber (Akki \& Haber, 1989; Akki, 1994), and was extended by Vatalaro and Forcella in (Vatalaro \& Forcella, 1997) to account for scattering in three dimensions (3-D), and by Linnartz and Fiesta in (Linnartz \& Fiesta, 1996) to include LOS scenarios. Some channel measurement results for narrowband IVC communications have been presented in (Kovacs et al., 2002; Maurer et al., 2002; Cheng et al., 2007). R.Wang and D.Cox (Wang \& Cox, 2002) introduced the discrete line spectrum method to simulate the IVC channels. Whereas the accuracy of this method was assured only for short-duration waveforms, Moreover, the numerical integrations required in determining the discrete set of frequencies and corresponding Doppler spectrum make the implementation complex and not easily reconfigurable for different Doppler frequencies or the Doppler frequency ratio. So it is not always suitable for real time hardware channel emulation or software simulation. A method based on inverse fast Fourier transform (IFFT) was presented by D.J.Young and N.C.Beaulieu (Young \& Beaulieu, 2000). This method was more accurate and efficient than the method of discrete line spectrum, but the IFFT-based method requires a complex elliptic integration. The authors in (Patel et al., 2003) proposed a "double-ring" scattering model to simulate the IVC scattering environment and developed modifications of two SoS models (statistical and deterministic SoS models) often used to simulate I2V channels in (Patel et al., 2005). More recently, Wang and Liu (Wang et al., 2009) presented a scattering Rician IVC fading model with a LOS component by SoS method, which is based on the Rayleigh model proposed in (Patel et al., 2005). A new statistical SoS in (Zajic \& Stuber, 2006) model is proposed for Rayleigh IVC fading channel to directly generate multiple uncorrelated complex envelope, which shows faster convergence than the model in (Patel et al., 2005) and adequate statistics with small simulation trials.

The statistical properties of Xiao and Zheng's simulators in (Xiao et al., 2006) are confirmed by extensive simulation results, showing good agreement with theoretical analysis in all cases and is a typical model with high quality for I2V channels. But with the development of mobile ad hoc networks, VANET channel modeling often involves the IVC channels, which is generally considered as the common case of the I2V channels. Therefore, in this chapter, we mainly focus on the modeling for IVC channels in VANETs. This motivates us to extend the new statistical SoS model in (Zajic \& Stuber, 2006) by employ a LOS component to characterize the IVC channels of VANETs. Furthermore, the deterministic SoS model, proposed in (Patel et al., 2005), are employed to simulate Rayleigh IVC channel for its reduced-complexity and theoretical and simulation results verified the usefulness of the model. Seeking to find a more suitable Rician simulation model for VANET channels, we also introduce a LOS component to extend the deterministic SoS model for comparison. 


\section{The mathematical reference model}

Akki and Haber's simulation model for IVC channels with no LOS component gives the complex channel envelope as (Akki \& Haber, 1989)

$$
Y(t)=\sqrt{\frac{2}{N}} \sum_{n=1}^{N} \exp \left\{j\left[\left(2 \pi f_{1} \cos \left(\alpha_{n}\right) t+2 \pi f_{2} \cos \left(\beta_{n}\right) t+\theta_{n}\right)\right]\right\}
$$

where $f_{1}$ and $f_{2}$ are the maximum Doppler frequencies due to the motion of the transmitter and the receiver, respectively. $N$ is the number of propagation paths, $\alpha_{n}$ and $\beta_{n}$ are the random angle of departure (AOD) and the angle of arrival (AOA) of the $n t h$ path measured with respect to the transmitter and the receiver velocity vectors, respectively, and $\theta_{n}$ is the random phase uniformly distributed on $[-\pi, \pi)$, independent of $\alpha_{n}^{\prime} s$ and $\beta_{n}^{\prime} s$ for all $n$.
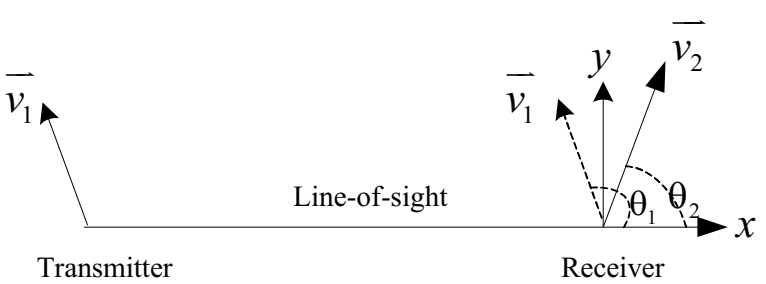

Fig. 1. IVC channel with a LOS component in VANETs

\section{Definitions:}

- In Fig. 1 , the velocities of transmitter and receiver are $\mathbf{v}_{1}, \mathbf{v}_{2}, \lambda$ is the carrier wavelength, $a=\left|\mathbf{v}_{1}\right| /\left|\mathbf{v}_{2}\right|$.

- $\theta_{1}, \theta_{2}$ are the angle between $\mathbf{v}_{1}, \mathbf{v}_{2}$ and the LOS component, respectively.

- $\mathbf{v}_{x}$ and $\mathbf{v}_{y}$ are the relative velocity of receiver to the transmitter in the $\mathrm{x}$-axis and $\mathrm{y}$-axis direction, respectively.

- the Doppler frequency caused by $\mathbf{v}_{x}$ and $\mathbf{v}_{y}$ are $f_{x}, f_{y}$.

The angle between $\mathbf{v}_{x}$ and LOS component is $0^{\circ}$ and the direction of $\mathbf{v}_{y}$ is perpendicular to the LOS component. From (Gregory, 2003), the Doppler frequency caused by LOS component in the IVC environment is $\left|f_{x}\right|=\left(\left|\mathbf{v}_{2}\right| \cos \theta_{2}-\left|\mathbf{v}_{1}\right| \cos \theta_{1}\right) / \lambda$. The LOS component is given by

$$
L=\sqrt{K} \exp \left[j\left\{2 \pi\left(\left|\mathbf{v}_{2}\right| \sin \theta_{2}-\left|\mathbf{v}_{1}\right| \sin \theta_{1}\right)\right\} t+\phi_{0}\right]
$$

where $K$ is the ratio of the specular power to the scattering power, and the initial phase $\phi_{0}$ is uniformly distributed over $[-\pi, \pi)$.

With reference to (1) and (2), the received complex envelope of the IVC fading channel with a LOS component can be expressed as

$$
Z(t)=\frac{Y(t)+\sqrt{K} \exp \left(j 2 \pi f_{0} t+\phi_{0}\right)}{\sqrt{1+K}}
$$

where $f_{0}=\left(\left|\mathbf{v}_{2}\right| \cos \theta_{2}-\left|\mathbf{v}_{1}\right| \cos \theta_{1}\right) / \lambda$.

Assuming omnidirectional antennas and isotropic scattering conditions around the transmitter and the receiver, the statistical properties of the reference model are given as follows. 
The auto-correlation and cross-correlation functions of the in-phase and quadrature components, and the auto-correlation functions of the complex envelope of fading signal $Z(t)$ are given by

$$
\begin{gathered}
R_{Z_{c} Z_{c}}(\tau)=R_{Z_{s} Z_{s}}(\tau)=\frac{2 J_{0}\left(2 \pi f_{1} \tau\right) J_{0}\left(2 \pi f_{2} \tau\right)+K \cos \left(2 \pi f_{0} \tau\right)}{2(1+K)} \\
R_{Z_{c} Z_{s}}(\tau)=-R_{Z_{s} Z_{c}}(\tau)=\frac{K \sin \left(2 \pi f_{0} \tau\right)}{2(1+K)} \\
R_{Z Z}(\tau)=\frac{2 J_{0}\left(2 \pi f_{1} \tau\right) J_{0}\left(2 \pi f_{2} \tau\right)+K \exp \left(j 2 \pi f_{0} \tau\right)}{(1+K)}=\frac{2 J_{0}\left(2 \pi a f_{2} \tau\right) J_{0}\left(2 \pi f_{2} \tau\right)+K \exp \left(j 2 \pi f_{0} \tau\right)}{(1+K)}
\end{gathered}
$$

where $J_{0}(\cdot)$ is the zeroth-order Bessel function of the first kind, $a=f_{1} / f_{2}$ is the ratio of two maximum Doppler frequencies (or vehicles speeds), and here assuming $f_{1} \leq f_{2}$. $a=0$ means that the transmitter is stationary and then equation (6) gives the auto-correlation for I2V channels, which indicates that I2V channels are a special case of IVC channels in VANETs.

Time-averaging is often used in place of ensemble averaging in simulation practice. The auto-correlation function of the real part of $Z(t)$ for one trial (sample of the process) then becomes

$$
\begin{aligned}
\hat{R}_{Z_{c} Z_{c}}(\tau) & =\lim _{T \rightarrow \infty} \frac{1}{T} \int_{0}^{T} Z_{c}(t) Z_{c}(t+\tau) d t \\
& =\frac{1}{N} \sum_{n=1}^{N} \cos \left(2 \pi f_{1} \cos \alpha_{n}+2 \pi f_{2} \cos \beta_{n}\right) \tau
\end{aligned}
$$

Furthermore, the time averaging changes from trial to trial due to the random angle. As pointed out in (Xiao et al., 2006), the variance of the time average $\operatorname{Var}[R(\cdot)]=E\left[|R(\cdot)-\hat{R}(\cdot)|^{2}\right]$ provides a measure of the closeness of the model in simulating the desired channel with a finite number of sinusoids. A lower variance indicates that a smaller number of simulation trials are needed to achieve the desired statistical properties, and, the convergence of the corresponding model is faster. The time-averaged variances of the aforementioned correlation statistics are derived as

$$
\begin{gathered}
\operatorname{Var}\left[R_{Z_{c} Z_{c}}(\tau)\right]=\operatorname{Var}\left[R_{Z_{s} Z_{s}}(\tau)\right]=\left[\frac{1+J_{0}\left(4 \pi a f_{2} \tau\right) J_{0}\left(4 \pi f_{2} \tau\right)-2 J_{0}^{2}\left(2 \pi a f_{2} \tau\right) J_{0}^{2}\left(2 \pi f_{2} \tau\right)}{2 N}\right] /(1+K)^{2} \\
\operatorname{Var}\left[R_{Z_{c} Z_{s}}(\tau)\right]=\operatorname{Var}\left[R_{Z_{s} Z_{c}}(\tau)\right]=\left[\frac{1-J_{0}\left(4 \pi a f_{2} \tau\right) J_{0}\left(4 \pi f_{2} \tau\right)}{2 N}\right] /(1+K)^{2} \\
\operatorname{Var}\left[R_{Z Z}(\tau)\right]=\operatorname{Var}\left[R_{Z Z}(\tau)\right]=\left[\frac{4-4 J_{0}^{2}\left(2 \pi a f_{2} \tau\right) J_{0}^{2}\left(2 \pi f_{2} \tau\right)}{N}\right] /(1+K)^{2}
\end{gathered}
$$

In next sections, we use these statistics to compare the performance of the proposed models with this reference model.

\section{Two SoS simulation models for VANETs}

This section proposes two Rician channel models with a LOS component for VANETs by introducing the aforementioned LOS component and extends two SoS models (statistical and deterministic simulation models). Important statistical properties for the proposed Rician models are derived and provided for comparison purposes. 


\subsection{Statistical SoS model}

Recently, A new model is proposed in (Zajic \& Stuber, 2006) to directly generate multiple uncorrelated complex envelope, which has resolved the difficulty in producing time averaged auto- and cross-correlation functions that match the reference model (Akki \& Haber, 1989).

The $k^{\text {th }}$ complex faded envelope is given by (Zajic \& Stuber, 2006)

$$
Y_{k}(t)=Y_{c k}(t)+j Y_{s k}(t)
$$

where

$$
\begin{aligned}
& Y_{c k}(t)=\frac{2}{\sqrt{N_{0} M}} \sum_{n=1}^{N_{0}} \sum_{m=1}^{M} \cos \left[2 \pi f_{2} t \cos \left(\beta_{m k}\right)\right] \cos \left[\left(2 \pi f_{1} t \cos \left(\alpha_{n k}\right)+\phi_{n m k}\right]\right. \\
& Y_{s k}(t)=\frac{2}{\sqrt{N_{0} M}} \sum_{n=1}^{N_{0}} \sum_{m=1}^{M} \sin \left[2 \pi f_{2} t \cos \left(\beta_{m k}\right)\right] \sin \left[\left(2 \pi f_{1} t \sin \left(\alpha_{n k}\right)+\phi_{n m k}\right]\right.
\end{aligned}
$$

$f_{1}, f_{2}, \alpha_{n k}, \beta_{m k}$ and $\phi_{n m k}$ are the maximum radian Doppler frequencies, the random angle of departure, the random angle of arrival, and the random phase, respectively. It is assumed that $P$ independent complex faded envelopes are required $(k=0, \ldots, P-1)$ each consisting of NM sinusoidal components. The angles of departures and the angles of arrivals are chosen as follows:

$$
\begin{gathered}
\alpha_{n k}=\frac{2 \pi n}{4 N_{0}}+\frac{2 \pi k}{4 P N_{0}}+\frac{\theta-\pi}{4 N_{0}} \\
\beta_{m k}=\frac{1}{2}\left(\frac{2 \pi m}{M}+\frac{2 \pi k}{P M}+\frac{\psi-\pi}{M}\right)
\end{gathered}
$$

where $n=1, \ldots, N_{0}, m=1, . . M, k=0, \ldots, P-1$. The angles of departures and the angles of arrivals in the $k^{\text {th }}$ complex faded envelope are obtained by rotating the angles of departures and the angles of arrivals in the $(k-1)^{t h}$ complex envelope by $2 \pi /\left(4 P N_{0}\right)$ and $2 \pi /(2 P M)$, respectively. The parameters $\phi_{n m k}, \theta$ and $\psi$ are independent random variables uniformly distributed on the interval $[-\pi, \pi)$.

With reference to (2),(11),(12),(13), the received complex envelope of the IVC fading channels can be obtained as follows:

$$
Z_{k}(t)=\frac{Y_{k}(t)+\sqrt{K} \exp \left(j 2 \pi f_{0} t+\phi_{0}\right)}{\sqrt{1+K}}
$$

The time-average auto-correlation and cross-correlation function of the in-phase and quadrature components, and the auto-correlation functions of the complex envelope of fading signal $Z_{k}(t)$ are given by

$$
\begin{gathered}
\hat{R}_{Z_{c k} Z_{c k}}(\tau)=\hat{R}_{Z_{s k} Z_{s k}}(\tau)=\frac{2 J_{0}\left(2 \pi f_{1} \tau\right) J_{0}\left(2 \pi f_{2} \tau\right)+K \cos \left(2 \pi f_{0} \tau\right)}{2(1+K)} \\
\hat{R}_{Z_{c k} Z_{s k}}(\tau)=-\hat{R}_{Z_{s k} Z_{c k}}(\tau)=\frac{K \sin \left(2 \pi f_{0} \tau\right)}{2(1+K)} \\
\hat{R}_{Z_{k} Z_{k}}(\tau)=\frac{2 J_{0}\left(2 \pi f_{1} \tau\right) J_{0}\left(2 \pi f_{2} \tau\right)+K \exp \left(j 2 \pi f_{0} \tau\right)}{1+K}
\end{gathered}
$$


Proof: we first prove the equation (17)

$$
\begin{aligned}
& R_{Z_{c k} Z_{c k}}(\tau) \\
& =E\left[Z_{c k}(t) Z_{c k}(t+\tau)\right] \\
& =\frac{1}{1+K}\left\{\frac { 4 } { N _ { 0 } M } E \left[\sum_{n, m=1}^{N_{0}, M} \cos \left(2 \pi f_{1} t \cos \alpha_{n k}+\phi_{n m k}\right) \cos \left(2 \pi f_{2} t \cos \beta_{m k}\right)\right.\right. \\
& \left.\cdot \sum_{p, q=1}^{N_{0}, M} \cos \left(2 \pi f_{1}(t+\tau) \cos \alpha_{p k}+\phi_{p q k}\right) \cos \left(2 \pi f_{2}(t+\tau) \cos \beta_{q k}\right)\right]+K E\left[\cos \left(2 \pi f_{0} t+\phi_{0}\right)\right. \\
& \left.\cdot \cos \left(2 \pi f_{0}(t+\tau)+\phi_{0}\right)\right]+2 \sqrt{\frac{K}{N_{0} M}} E\left[\sum_{n, m=1}^{N_{0}, M} \cos \left(2 \pi f_{1} t \cos \alpha_{n k}+\phi_{n m k}\right) \cos \left(2 \pi f_{2} t \cos \beta_{m k}\right)\right. \\
& \left.\cdot \cos \left(2 \pi f_{0}(t+\tau)+\phi_{0}\right)\right]+E\left[\cos \left(2 \pi f_{0} t+\phi_{0}\right)\right. \\
& \left.\left.\cdot 2 \sqrt{\frac{K}{N_{0} M}} \sum_{n, m=1}^{N_{0}, M} \cos \left(2 \pi f_{1}(t+\tau) \cos \alpha_{n k}+\phi_{n m k}\right) \cos \left(2 \pi f_{2}(t+\tau) \cos \beta_{m k}\right)\right]\right\} \\
& \left.=\frac{1}{1+K}\left\{\frac{1}{N_{0} M} E\left[\sum_{n, m=1}^{N_{0}, M} \cos \left(2 \pi f_{1} \tau \cos \alpha_{n k}\right) \cos \left(2 \pi f_{2} \tau \cos \beta_{m k}\right)\right]\right\}+\frac{K}{2(1+K)} \cos \left(2 \pi f_{0} \tau\right)\right\} \\
& =\frac{1}{1+K}\left\{\frac{1}{N_{0}} \sum_{n=1}^{N_{0}} \frac{1}{2 \pi} \int_{-\pi}^{\pi} \cos \left[2 \pi f_{1} \tau \cos \left(\frac{2 \pi n}{4 N_{0}}+\frac{2 \pi k}{4 P N_{0}}+\frac{\theta-\pi}{4 N_{0}}\right)\right] d \theta\right. \\
& \left.\cdot \frac{1}{M} \sum_{m=1}^{M} \frac{1}{2 \pi} \int_{-\pi}^{\pi} \cos \left[2 \pi f_{2} \tau \cos \left(\frac{2 \pi m}{2 M}+\frac{2 \pi k}{2 P M}+\frac{\psi-\pi}{2 M}\right)\right] d \psi\right\}+\frac{K}{2(1+K)} \cos \left(2 \pi f_{0} \tau\right) \\
& =\frac{1}{1+K}\left\{\frac{1}{N_{0}} \sum_{n=1}^{N_{0}} \frac{1}{2 \pi} \int_{\frac{2 \pi(n-1)}{4 N_{0}}+\frac{2 \pi k}{4 P N_{0}}}^{\frac{2 \pi n}{4 N_{0}}+\frac{2 \pi k}{4 N_{0}}} \cos \left(2 \pi f_{1} \tau \cos \gamma_{n}\right) \cdot 4 N_{0} d \gamma_{n}\right. \\
& \left.\cdot \frac{1}{M} \sum_{n=1}^{M} \frac{1}{2 \pi} \int_{\frac{2 \pi(m-1)}{2 M}+\frac{2 \pi k}{2 P M}}^{\frac{2 \pi M}{2 M}+\frac{2 \pi k}{2 M}} \cos \left(2 \pi f_{2} \tau \cos \gamma_{m}\right) \cdot 2 M d \gamma_{m}\right\}+\frac{K}{2(1+K)} \cos \left(2 \pi f_{0} \tau\right) \\
& =\frac{1}{1+K}\left\{\frac{1}{N_{0}} \cdot \frac{1}{2 \pi} \int_{\frac{2 \pi k}{4 P N_{0}}}^{\frac{\pi}{2}+\frac{2 \pi k}{4 P N_{0}}} \cos \left(2 \pi f_{1} \tau \cos \gamma_{n}\right) \cdot 4 N_{0} d \gamma_{n}\right. \\
& \left.\cdot \frac{1}{M} \cdot \frac{1}{2 \pi} \int_{\frac{2 \pi k}{2 P M}}^{\pi+\frac{2 \pi k}{2 P M}} \cos \left(2 \pi f_{2} \tau \cos \gamma_{m}\right) \cdot 2 M d \gamma_{m}\right\}+\frac{K}{2(1+K)} \cos \left(2 \pi f_{0} \tau\right) \\
& =\frac{1}{1+K}\left[\frac{2}{\pi} \int_{0}^{\frac{\pi}{2}} \cos \left(2 \pi f_{1} \tau \cos \gamma_{1}\right) d \gamma_{1} \cdot \frac{1}{\pi} \int_{0}^{\pi} \cos \left(2 \pi f_{2} \tau \cos \gamma_{2}\right) d \gamma_{2}\right] \frac{K}{2(1+K)} \cos \left(2 \pi f_{0} \tau\right) \\
& =\frac{2 J_{0}\left(2 \pi f_{1} \tau\right) J_{0}\left(2 \pi f_{2} \tau\right)+K \cos \left(2 \pi f_{0} \tau\right)}{2(1+K)}
\end{aligned}
$$


This completes the proof of (17). Similarly, one can prove the (18) and (19), details are omitted for brevity.

The time-averaged variances of above correlation statistics of $Z_{k}(t)$ are presented as

$$
\begin{aligned}
& \operatorname{Var}\left\{\hat{R}_{Z_{c k} Z_{c k}}(\tau)\right\}= \operatorname{Var}\left\{\hat{R}_{Z_{s k} Z_{s k}}(\tau)\right\} \\
&= {\left[\frac{1+J_{0}\left(4 \pi f_{1} \tau\right) J_{0}\left(4 \pi f_{2} \tau\right)}{4 N_{0} M}-f_{c}\left(2 \pi f_{1} \tau, 2 \pi f_{2} \tau\right)\right] /(1+K)^{2} } \\
& \operatorname{Var}\left\{\hat{R}_{\left.Z_{c k} Z_{s k}(\tau)\right\}=\operatorname{Var}\left\{\hat{R}_{Z_{s k} Z_{c k}}(\tau)\right\}=0}\right. \\
& \operatorname{Var}\left\{\hat{R}_{Z Z}(\tau)\right\}=\left[\frac{1+J_{0}\left(4 \pi f_{1} \tau\right) J_{0}\left(4 \pi f_{2} \tau\right)}{N_{0} M}-4 f_{c}\left(2 \pi f_{1} \tau, 2 \pi f_{2} \tau\right)\right] /(1+K)^{2}
\end{aligned}
$$

where

$$
\begin{aligned}
f_{c}\left(2 \pi f_{1} \tau, 2 \pi f_{2} \tau\right)= & \sum_{k_{1}=1}^{N_{0}}\left[\frac{1}{2 \pi} \int_{\frac{2 \pi\left(k_{1}-1\right)}{N}+\frac{2 \pi k}{4 P N_{0}}}^{\frac{2 \pi k_{1}}{4 P N_{0}}} \cos \left(2 \pi f_{1} \tau \cos \gamma_{1}\right) d \gamma_{1}\right]^{2} . \\
& \sum_{k_{2}=1}^{M}\left[\frac{1}{2 \pi} \int_{\frac{2 \pi\left(k_{2}-1\right)}{M}+\frac{2 \pi k}{2 P M}}^{\frac{2 \pi k_{2}}{M}+\frac{2 \pi k}{M}} \cos \left(2 \pi f_{2} \tau \cos \gamma_{2}\right) d \gamma_{2}\right]^{2}
\end{aligned}
$$

Proof: We start with the first part of (20) and derive

$$
\begin{aligned}
& \operatorname{Var}\left\{\hat{R}_{Z_{c k} Z_{c k}}(\tau)\right\} \\
&=E\left[\left|\hat{R}_{Z_{c k} Z_{c k}}(\tau)-\frac{2 J_{0}\left(2 \pi f_{1} \tau\right) J_{0}\left(2 \pi f_{2} \tau\right)+K \cos \left(2 \pi f_{0} \tau\right)}{2(1+K)}\right|^{2}\right] \\
&=E\left[\left|\hat{R}_{Z_{c k} Z_{c k}}(\tau)\right|^{2}\right]-\frac{J_{0}^{2}\left(2 \pi f_{1} \tau\right) J_{0}^{2}\left(2 \pi f_{2} \tau\right)}{(1+K)^{2}}-\left[\frac{K \cos 2 \pi f_{0} \tau}{2(1+K)}\right]^{2} \\
&=E\left\{\frac { 1 } { ( 1 + K ) ^ { 2 } } \cdot \frac { 1 } { N _ { 0 } ^ { 2 } M ^ { 2 } } \left[\sum_{n, m=1}^{N_{0}, M} \cos \left(2 \pi f_{1} \tau \cos \alpha_{n k}\right) \cos \left(2 \pi f_{2} \tau \cos \beta_{m k}\right)\right.\right. \\
&\left.\left.\cdot \sum_{p, q=1}^{N_{0}, M} \cos \left(2 \pi f_{1} \tau \cos \alpha_{p k}\right) \cos \left(2 \pi f_{2} \tau \cos \beta_{q k}\right)\right]\right\}-\frac{J_{0}^{2}\left(2 \pi f_{1} \tau\right) J_{0}^{2}\left(2 \pi f_{2} \tau\right)}{(1+K)^{2}} \\
&= \frac{1}{(1+K)^{2}} \cdot \frac{1}{N_{0}^{2} M^{2}}\left\{E\left[\sum_{n, m=1}^{N_{0}, M} \cos { }^{2}\left(2 \pi f_{1} \tau \cos \alpha_{n k}\right) \cos { }^{2}\left(2 \pi f_{2} \tau \cos \beta_{m k}\right)\right]\right. \\
& \cdot \sum_{n, m=1}^{N_{0}, M} \sum_{p, q=1}^{N_{0}, M} E\left[\cos \left(2 \pi f_{1} \tau \cos \alpha_{n k}\right) \cos \left(2 \pi f_{2} \tau \cos \beta_{m k}\right)\right] E\left[\cos \left(2 \pi f_{1} \tau \cos \alpha_{p k}\right)\right. \\
&\left.\left.\cdot \cos \left(2 \pi f_{2} \tau \cos \beta_{q k}\right)\right]\right\}-\frac{J_{0}^{2}\left(2 \pi f_{1} \tau\right) J_{0}^{2}\left(2 \pi f_{2} \tau\right)}{(1+K)^{2}} \quad(n \neq p \quad \text { or } \quad m \neq q) \\
&= \frac{1}{(1+K)^{2}} \cdot \frac{1}{N_{0}^{2} M^{2}}\left\{N_{0} M \cdot \frac{1+J_{0}\left(4 \pi f_{1} \tau\right) J_{0}\left(4 \pi f_{2} \tau\right)}{4}\right. \\
&\left.+N_{0}^{2} M^{2}\left[J_{0}^{2}\left(2 \pi f_{1} \tau\right) J_{0}^{2}\left(2 \pi f_{2} \tau\right)-f_{c}\left(2 \pi f_{1} \tau, 2 \pi f_{2} \tau\right)\right]\right\}-\frac{J_{0}^{2}\left(2 \pi f_{1} \tau\right) J_{0}^{2}\left(2 \pi f_{2} \tau\right)}{(1+K)^{2}} \\
&= {\left[\frac{1+J_{0}\left(4 \pi f_{1} \tau\right) J_{0}\left(4 \pi f_{2} \tau\right)}{4 N_{0} M}-f_{c}\left(2 \pi f_{1} \tau, 2 \pi f_{2} \tau\right)\right] /(1+K)^{2} }
\end{aligned}
$$


Similarly, we can validate the second part of (20) and equation (21). Thus, we have

$$
\begin{aligned}
& \operatorname{Var}\left\{\hat{R}_{Z_{k} Z_{k}}(\tau)\right\} \\
& =E\left[\left|\hat{R}_{Z_{k} Z_{k}}(\tau)-\frac{2 J_{0}\left(2 \pi f_{1} \tau\right) J_{0}\left(2 \pi f_{2} \tau\right)+K \exp \left(j 2 \pi f_{0} \tau\right)}{1+K}\right|^{2}\right] \\
& =E\left[\left|2 \hat{R}_{Z_{c k} Z_{c k}}(\tau)+j 2 \hat{R}_{Z_{c k} Z_{s k}}(\tau)-\frac{2 J_{0}\left(2 \pi f_{1} \tau\right) J_{0}\left(2 \pi f_{2} \tau\right)}{(1+K)}-\frac{K \exp j 2 \pi f_{0} \tau}{(1+K)}\right|\right]^{2} \\
& =\left[\frac{1+J_{0}\left(4 \pi f_{1} \tau\right) J_{0}\left(4 \pi f_{2} \tau\right)}{N_{0} M}-4 f_{c}\left(2 \pi f_{1} \tau, 2 \pi f_{2} \tau\right)\right] /(1+K)^{2}
\end{aligned}
$$

This completes the proof.

\subsection{Deterministic SoS model}

The model proposed above may require several simulation trials to converge to the desired properties. A low-complexity alternative is described in this section. It was recently used for IVC channels with no LOS component (Patel et al., 2005) and called the MEDS model. The complex faded envelope generated by the MEDS model is given by

$$
\begin{gathered}
Y(t)=Y_{c}(t)+j Y_{s}(t) \\
Y_{c}(t)=\sqrt{\frac{2}{N_{c} M_{c}}} \sum_{n, m=1}^{N_{c}, M_{c}} \cos \left(2 \pi f_{1, n}^{c} t+2 \pi f_{2, m}^{c} t+\phi_{n m}^{c}\right) \\
Y_{s}(t)=\sqrt{\frac{2}{N_{s} M_{s}}} \sum_{n, m=1}^{N_{s}, M_{s}} \cos \left(2 \pi f_{1, n}^{s} t+2 \pi f_{2, m}^{s} t+\phi_{n m}^{s}\right) \\
f_{1, n}^{c / s}=f_{1} \cos \left(\frac{\pi(n-0.5)}{2 N_{c / s}}\right) \quad n=1,2, \ldots N_{c / s} \\
f_{2, m}^{c / s}=f_{2} \cos \left(\frac{\pi(m-0.5)}{M_{c / s}}\right) \quad m=1,2, \ldots M_{c / s}
\end{gathered}
$$

where the phase $\phi_{n m} \sim U[-\pi, \pi)$ is independent for all $n, m$ and the in-phase and quadrature components.

Similarly, with reference to $(2),(25),(26),(27),(28)$, the complex signal of the IVC channel model are expressed as

$$
Z(t)=\frac{Y(t)+\sqrt{K} \exp \left(j 2 \pi f_{0} t+\phi_{0}\right)}{\sqrt{1+K}}
$$

As described in (Patel et al., 2005), all the frequencies, $f_{1, n^{\prime}}^{c} f_{2, m}^{c}$ and $f_{1, k^{\prime}}^{s} f_{2, l}^{s}$ must be distinct. In addition, $f_{1, n^{\prime}}^{c} f_{2, m}^{c}$ and $f_{1, k}^{c}, f_{2, l}^{c}$ have also to be distinct. From simulations, we found that with $N_{c}=M_{c}=N_{C}$ and $N_{S}=M_{S}=N_{C}+1$, the Doppler frequencies are indeed distinct for practical ranges varying from 5 to 60 . Under these assumptions, it can be shown that the time-average correlations are equal to the statistical correlations. 


$$
\begin{gathered}
\hat{R}_{Z_{c} Z_{c}}(\tau)=\frac{1}{1+K}\left[\frac{1}{N_{C}^{2}} \sum_{n, m=1}^{N_{c}, N_{c}} \cos \left\{2 \pi f_{1, n}^{c} \tau+2 \pi f_{2, m}^{c} \tau\right\}+\frac{K \cos \left(2 \pi f_{0} \tau\right)}{2}\right] \\
\hat{R}_{Z_{s} Z_{s}}(\tau)=\frac{1}{1+K}\left[\frac{1}{\left(N_{C}+1\right)^{2}} \sum_{n, m=1}^{N_{c}+1, N_{c}+1} \cos \left\{2 \pi f_{1, n}^{s} \tau+2 \pi f_{2, m}^{s} \tau\right\}+\frac{K \cos \left(2 \pi f_{0} \tau\right)}{2}\right] \\
\hat{R}_{Z_{c} Z_{s}}(\tau)=-\hat{R}_{Z_{s} Z_{c}}(\tau)=\frac{K \sin \left(2 \pi f_{0} \tau\right)}{2(1+K)} \\
\hat{R}_{Z Z}(\tau)=\frac{1}{1+K}\left[\frac{1}{N_{C}^{2}} \sum_{n, m=1}^{N_{c}, N_{c}} \cos \left\{2 \pi f_{1, n}^{c} \tau+2 \pi f_{2, m}^{c} \tau\right\}+\right. \\
\left.\frac{1}{\left(N_{C}+1\right)^{2}} \sum_{n, m=1}^{N_{c}+1, N_{c}+1} \cos \left\{2 \pi f_{1, n}^{s} \tau+2 \pi f_{2, m}^{s} \tau\right\}+K \exp \left(2 \pi f_{0} \tau\right)\right]
\end{gathered}
$$

Remark: The expressions for variances of the correlation functions for the proposed MEDS model cannot be obtained in a simplified form, here are not provided.

\section{Performance analysis and comparison}

This section compares the performance of the proposed simulation models. Unless stated otherwise, all the simulation results presented here are obtained using a normalized sampling period $f_{1} T_{S}=0.01\left(T_{S}\right.$ is the sampling period).

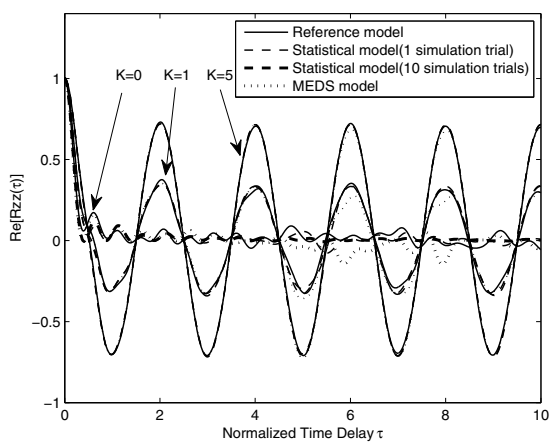

(a)

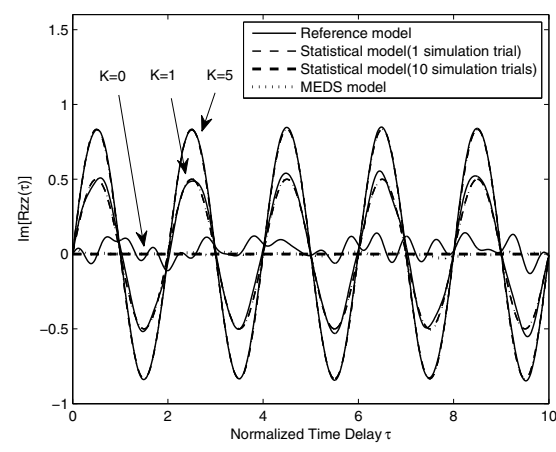

(b)

Fig. 2. The auto-correlation function of the complex envelope with different $K$

\subsection{Effects of Rician factor in VANETs}

The results of Figs. 2-3 are obtained using $a=1, f_{1}=f_{2}=50 \mathrm{~Hz}, N_{0}=M=N_{C}=P=8$. For a fair comparison, we use $N=4 N_{0} \times 2 M=512$ sinusoids for simulation of the reference model. 


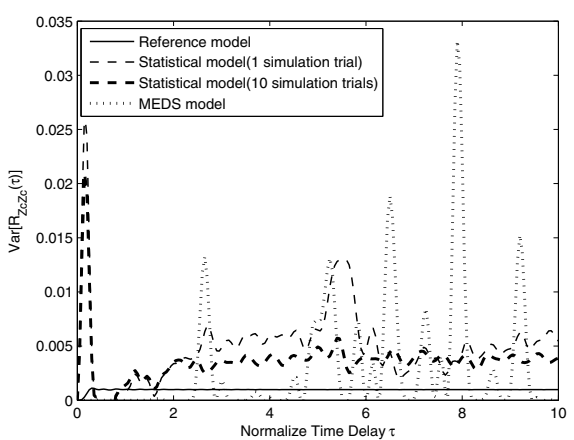

(a) $\mathrm{K}=0$

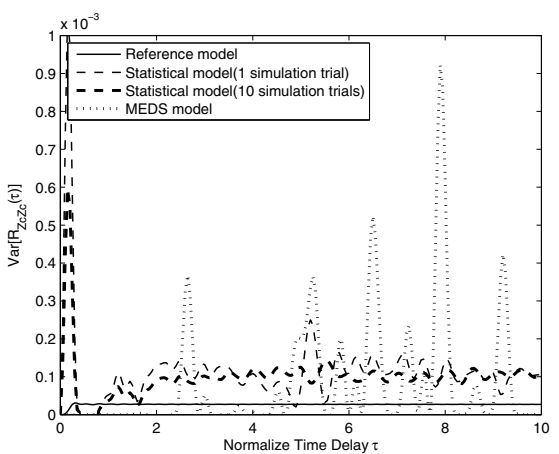

(c) $\mathrm{K}=5$

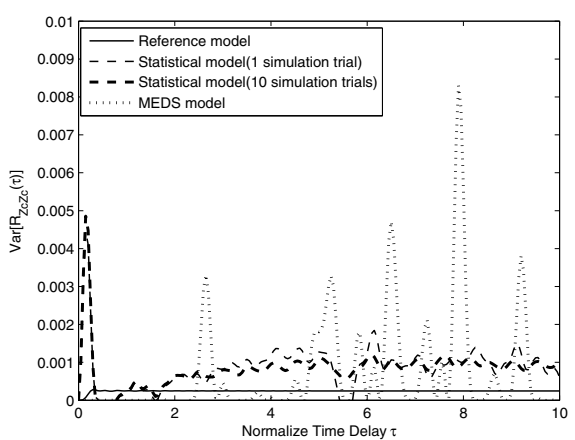

(b) $\mathrm{K}=1$

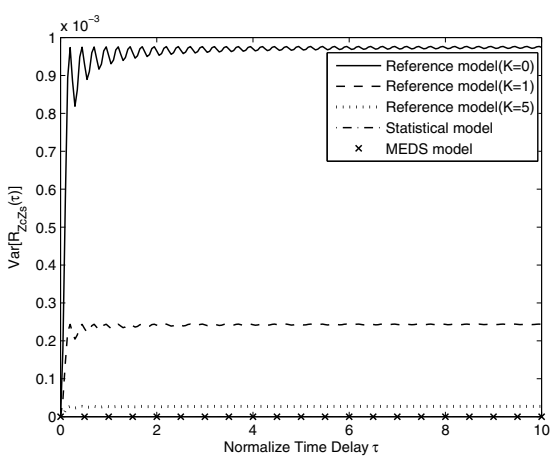

(d) Variance of the cross-correlation function

Fig. 3. Variance of the auto-correlation and cross-correlation function with different $K$

Fig. 2 shows the correlation properties of the aforementioned models with different $K$ factors in VANETs. For a large range of normalized time-delay $\left(0 \leq f_{1} T_{S} \leq 4\right)$, the proposed simulation models keep good agreement with the reference model, without exhibiting any sort of periodicity as encountered in Wang and Cox's model (Wang \& Cox, 2002). For the same time delay $\tau$, the magnitude of the channel correlation tends to be larger. As the $K$ factor increases, the proposed models get closer to the reference model.

Fig. 3 compares the variances of the auto- and cross-correlation functions for the proposed simulation models. As shown in Figs. 3a-3c, the variances of the auto-correlation functions decrease as $K$ factor increases. It indicates that the simulation models perform better under a larger amount of LOS components. When $K$ is larger, the LOS components become more dominant over the scattering components, which avoids the deviation caused by the finite scatters. We can also observe that the variances of the auto-correlation of our models are higher than the reference model. It is noted that the difference between the statistical model and the reference model becomes smaller when the number of the simulation trials is increased. Simulation results show that the statistical model achieves better convergence by averaging 10 simulation trials. Fig. $3 \mathrm{~d}$ shows that the variances of the cross-correlation for the proposed models are lower than the reference model. 


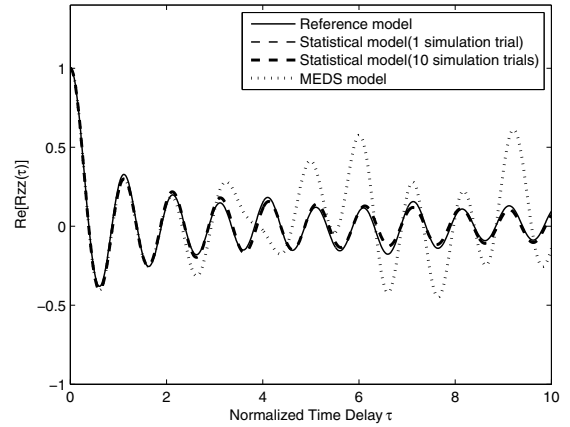

(a) $\mathrm{a}=0$

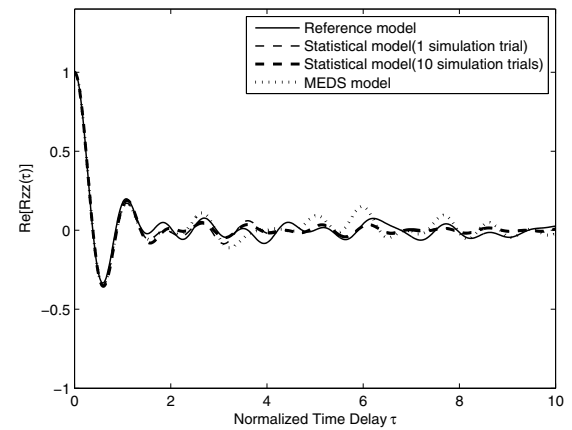

(b) $\mathrm{a}=0.2$

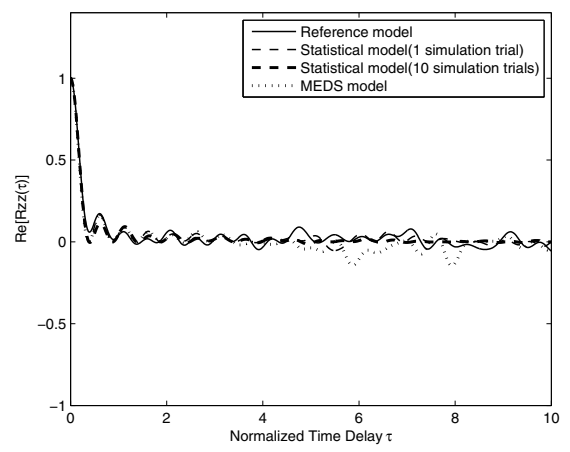

(c) $\mathrm{a}=1$

Fig. 4. Real part of the auto-correlation function of the complex envelope with different $a$

It should be noted that the plot of the auto-correlation function when $K=0$ keeps agreement with (Patel et al., 2005) and the result of the variance of auto-correlation is almost similar to that in (Zajic \& Stuber, 2006), which indicate that our models and performance analysis are more comprehensive than the existing ones.

\subsection{Effects of vehicle speed ratio in VANETs}

The simulation results presented in Figs. 4-5 are obtained using $K=0, f_{2}=50 \mathrm{~Hz}, f_{1}=0,10$ and $50 \mathrm{~Hz}$ when the corresponding value of speed ratio $a$ equals to $0,0.2$ and 1 . The imaginary part of the auto-correlation of the complex envelope for the proposed two SoS models is always equal to 0 , which is in line with the ideal situation and shows better performance compared with the reference model.

Fig. 4 shows the real part of correlation properties of the above models with different $a$ in VANETs. It is observed that the proposed models provide a better approximation to the desired auto-correlation when $a$ increases. From Figs. 5a-5c, we found that the variances of the auto-correlation of our models tend to be lower with a larger value of $a$. So the proposed models perform better with a smaller relative speed difference. A physical interpretation 


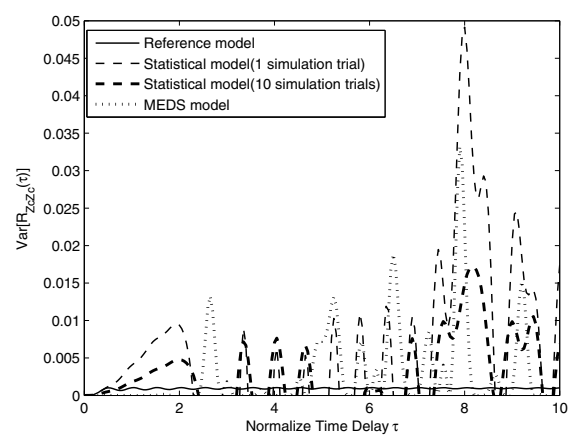

(a) $\mathrm{a}=0$

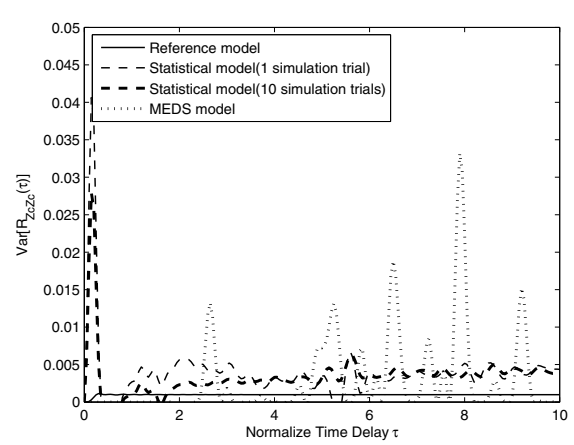

(c) $\mathrm{a}=1$

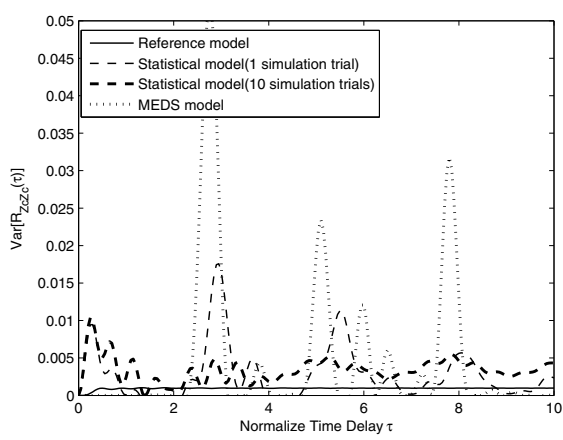

(b) $\mathrm{a}=0.2$

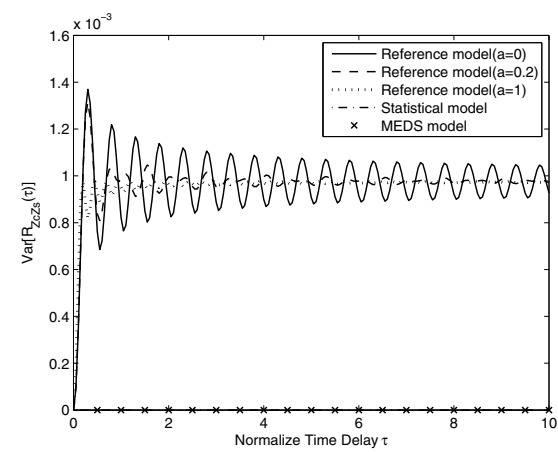

(d) Variance of the cross-correlation function

Fig. 5. Variance of the auto-correlation and cross-correlation function with different $a$

should be like this: smaller relative difference means that the transmitter and the receiver are in a relatively closer static state, so the randomness of channel tends to be smaller. As plotted in Fig. $5 \mathrm{~d}$, the variances of the cross-correlation of the proposed models are considerably lower than the reference model.

It should also be mentioned that our simulation results of variance of the correlation when $a=0$ represent the case of I2V channels, which shows the comprehensiveness of our models and performance analysis as well.

\section{Conclusion}

The automotive industry conveniently built powerful and safer cars by embedding advanced materials and sensors. With the advent of wireless communication technologies, cars are being equipped with wireless communication devices, enabling them to communicate with others. Such communications are not plainly restricted to data transfers (such as GPS, video and audio, emails, etc.), but also create new opportunities for enhancing road safety. Some applications only require communication among vehicles, while many others require the coordination between vehicles and road-side infrastructure. Recent technological developments, notably in mobile computing, wireless communication, and 
remote sensing are pushing ITS towards a major leap forward. Vehicles become sophisticated computing systems, with several computers and sensors onboard, each dedicated to certain car operations. Interconnected vehicles do not only collect information about themselves and their environments, but they also exchange the information in real time with other nearby vehicles. As radio-communication-based solutions can operate beyond the line-of-sight constraints, they can enable cooperative approaches. Vehicles and infrastructure cooperate to perceive potentially dangerous situations in an extended space and time horizon. Appropriate vehicular communication architectures are necessary to create reliable and extended driving support systems for road safety and transportation efficiency.

A number of technical challenges need to be resolved in order to deploy vehicular networks and to provide related services for drivers and passengers in such networks. Scalability and interoperability are two important issues that should be addressed. The employed protocols and mechanisms should be scalable to numerous vehicles and interoperable with different wireless technologies, such as reliable link performance and MAC protocols, routing and dissemination, IP configuration and mobility management, security etc. As a key component of the ITS, vehicular wireless networks, has attracted research attention from both the academia and industry of US, EU, and Japan. Although many works have been done on communication and routing protocol, only few models have been developed to characterize the fading effect in vehicular wireless network.

In this chapter, we proposed a new statistical and deterministic SoS model for IVC fading channels with a LOS component in VANETs. The properties of the proposed models were derived and verified in terms of the auto-correlation and the cross-correlation by comparison between theoretical and simulation results. The statistics of them match those of the reference model for a large range of normalized time delays $\left(0 \leq f_{1} T_{S} \leq 4\right)$. When the $K$ factor increases, the proposed SoS models show an improved approximation of the desired auto-correlation and faster convergence. And then we described the curves of the statistics for the simulation models with different vehicle speed ratios, which indicates that the smaller relative speed difference of two vehicle speeds in VANETs contribute to the better performance of the proposed models. More importantly, Based on our models, we provided more comprehensive performance analysis and comparison compared to existing models.

It is observed that the the variances in the cross-correlation for the statistical model and the MEDS model are considerably lower than those of reference model. Meanwhile, For the same time delay $\tau$, the statistical model shows better performance and faster convergence than the MEDS model. Hence, the statistical model may be more suitable for IVC fading channels with a LOS component in VANETs.

\section{Acknowledgments}

The authors would like to thank the Cognitive Radio Sensor Network research group in Electronic Information Engineering of Nanchang University for continuous support and lively discussions. The authors are also grateful to the anonymous reviewers for their helpful comments.

This work has been supported by the National Natural Science Foundation of China (No.60762 005), the Natural Science Foundation of Jiangxi Province for Youth (No.2010GQS0153 and No.2009GQS0070) and the Graduate Student Innovation Foundation of Jiangxi Province (No.YC10A032). 


\section{References}

M. Boban, O. K. Tonguz \& J. Barros (2009). Unicast Communication in Vehicular Ad Hoc Networks: A Reality Check, IEEE Commun. Lett., Vol. 13, No. 12, Dec. 2009, 995-997.

T. L. Willke, P. Tientrakool \& N. F.Maxemchuk (2009). A Survey of Inter-Vehicle Communication Protocols and Their Applications, IEEE Commun. Surv. \& Tuto., Vol. 11, No. 2, Jun. 2009, 3-20.

A. F. Molisch, F.Tufvesson, J.Karedal \& C.F.Mecklenbrauker (2009). A Survey on Vehicle-to-Vehicle Propagation Channels, IEEE Trans. Wireless Commun., Vol.16, No.6, Dec. 2009, 12-22.

A. M. Abbas \& O.Kure (2010). Quality of Service in mobile ad hoc networks: a survey, Int. J. Ad Hoc and Ubiquitous Computing, Vol. 6, No.2, Jul. 2010, 75-98.

T. Lwinmuller, E. Schoch \& F. Kargl (2006). Postion Verification Approaches for Vechicular Ad Hoc Networks, IEEE Trans.Commun., Vol. 13, No. 5, Jan. 2006, 278-285.

T. Taleb, E. Sakhaee, A. Jamalipour, K. Hashimoto, N. Kato \& Y. Nemoto (2007). A Stable Routing Protocol to Support ITS Services in VANET Networks, IEEE Trans. Veh. Technol., Vol. 56. No. 6, Nov. 2007, 3337-3347.

D. Kim, J.J. Garcia-Luna-Aceves, K. Obraczka, J. C. Cano \& P. Manzoni (2003). Routing Mechanisms for Mobile Ad Hoc Networks Based on the Energy Drain Rate, IEEE Trans. on mobile computing, Vol. 2, No. 2, April-June. 2003, 161-173.

X. J. Du \& D. P. Wu (2007). Adaptive Cell Relay Routing Protocol for Mobile Ad Hoc Networks IEEE Trans. Veh. Technol., Vol. 55. No. 1, Nov. 2007, 3381-3396.

H. Y. Huang, P. E. Luo, M. L. Li, D. Li, X. Li, W. Shu \& M. Y. Wu (2007). Performance Evaluation of SUVnet With Real-Time Traffic Data, IEEE Trans. Veh.Technol., Vol. 56. No. 6,Nov. 2007, 3381-3396.

N. Wisitpongphan, F. Bai, P. Mudalige, V. Sadekar, \& O. Tonguz (2007). Routing in Sparse Vehicular Ad Hoc Wireless Networks, IEEE J. Sel. Areas in Commun., Vol. 25, No. 8, Oct. 2007, 1538-1556.

J. Nzouonta, N. Rajgure \& G. Wang (2009). VANET Routing on City Roads Using Real-Time Vehicular Traffic Information, IEEE Trans Veh. Technol., Vol. 58, No. 7, Sep. 2009, 3609-3626.

W. J. Wang, F. Xie \& M. Chatterjee (2009). Small-Scale and Large-Scale Routing in Vehicular Ad Hoc Networks, IEEE Trans Veh. Technol., Vol. 58, No. 9, Nov. 2009, 5200-5213.

H. Saleet, O. Basir, R. Langar \& R. Boutaba (2010). Region-Based Location-Service-Management Protocol for VANETs, IEEE Trans Veh. Technol., Vol. 59, No. 2, Feb. 2010, 917-931.

V. Tarokh (2009). In: New Directions in wireless Communications Research, Springer Dordrecht Heidelberg London New York, 2009, 1-25.

C. S. Patel, G.L.Stuber \& Thomas G.Pratt (2005). Simulation of Rayleigh-faded mobile-to-mobile communication channels, IEEE Trans. Commun., Vol. 53, No. 11, Nov. 2005, 1876-1884.

A. G. Zajic \& G. L. Stuber (2006). A New Simulation Model for Mobile-to-Mobile Rayleigh Fading Channels, in IEEE Wireless Commun. Netw. Conf., (WCNC'06), pp. 1266-1270, Apr. 2006.

L. C. Wang, W. C. Liu \& Y. H. Cheng (2009). Statisitical Analysis of a Mobile-to-Mobile Rician Fading Channel Model, IEEE Trans. Veh. Technol., Vol. 58, No. 1, Jan. 2009, 32-38.

R. H. Clarke (1968). A statistical theory of mobile-radio reception, Bell Syst. Tech. J., Aug. 1968, 957-1000. 
M. J. Gans (1972). A power-spectral theory of propagation in the mobile-radio environment, IEEE Trans. Veh. Technol., Vol. 21, No. 1, Feb. 1972, 27-38.

W. C. Jakes (1994). Microwave Mobile Communications. Wiley, 1974; re-issued by IEEE Press, 1994.

P. Dent, G. E. Bottomley \& T. Croft (1993). Jakes fading model revisited, IEEE Electron. Lett., Vol. 29, No. 13, Jun. 1993, 1162-1163.

M. Patzold, U. Killat, F. Laue, \& Y. C. Li (1998). On the statistical properties of deterministic simulation models for mobile fading channels, IEEE Trans. Veh. Technol., Vol. 47, No. 1, Feb. 1998, 254-269.

K.-W. Yip and T.-S. Ng, "A simulation model for Nakagami-m fading channels, $\mathrm{m}<1$," IEEE Trans. Commun., vol. 48, No. 2, Feb.2000, 214-221.

M. F. Pop \& N. C. Beaulieu (2001). Limitations of sum-of-sinusoids fading channel simulators, IEEE Trans. Commun., Vol. 49, No. 4, Apr. 2001, 699-708.

Y. X. Li \& X. Huang (2002). The generation of independent Rayleigh faders, in Proc. IEEE ICC, pp. 41-45, Jun. 2000.

C. Xiao \& Y. R. Zheng (2002). A generalized simulation model for Rayleigh fading channels with accurate second-order statistics, in Proc. IEEE VTC-Spring, pp. 170-174, May 2002.

C. Xiao, Y. R. Zheng, \& N. C. Beaulieu (2002). Second-order statistical properties of the WSS Jakesą́r fading channel simulator, IEEE Trans. Commun., Vol. 50, No.6, Jun. 2002, 888-891.

Y. R. Zheng \& C. Xiao (2002). Improved models for the generation of multiple uncorrelated Rayleigh fading waveforms, IEEE Commun. Lett., Vol. 6, No.6, Jun. 2002, 256-258.

Y. R. Zheng \& C. Xiao (2003). Simulation models with correct statistical properties for Rayleigh fading channels, IEEE Trans. Commun., Vol. 51, No.6, Jun. 2003, 920-928.

C.Xiao, Y.R.Zheng \& N.C.Beaulieu (2006). Novel sum-of-sinusoids simulation models for Rayleigh and Rician fading channels, IEEE Trans. Wireless Commun., Vol.5, No.12, Dec. 2006, 3667-3679.

A. S. Akki \& F. Haber (1989). A statistical model for mobile-to-mobile land communication channel, IEEE Trans. Veh. Technol., Vol. 35, No. 1, Feb. 1986, 2-7.

A. S. Akki (1994). Statistical properties of mobile-to-mobile land communication channels, IEEE Trans. Veh. Technol., Vol. 43, No. 4, Nov. 1994, 826-831.

F. Vatalaro \& A. Forcella (1997). Doppler spectrum in mobile-to-mobile communications in the presence of three-dimensional multipath scattering, IEEE Trans. Veh. Technol., Vol. 46, No. 1, Feb. 1997, 213-219.

J. M. G. Linnartz \& R. F. Fiesta (1996). Evaluation of radio links and networks. [Online].Available: http://www.path.berkeley.edu/PATH/ Publications/PDF/PRR/96/ PRR-96-16.pdf.

I. Z. Kovacs, P. C. F. Eggers, K. Olesen \& L. G. Petersen (2002). Investigations of outdoor-to-indoor mobile-to-mobile radio communication channels, in Proc. IEEE Veh. Technol. Conf., Vancouver, BC, Canada, pp. 430-434, Sep. 2002.

J. Maurer, T. Fugen, K. Olesen \& W. Wiesbeck (2002). Narrowband measurement and analysis of the intervehicle transmission channel at $5.2 \mathrm{GHz}$, in Proc. IEEE Veh. Technol. Conf., Birmingham, AL, pp. 1274-1278, May 2002.

L. Cheng, B. E. Henty, D. D. Stancil, F. Bai \& P. Mudalige (2007). Mobile Vehicle-to-Vehicle Narrow-Band Channel Measurement and Characterization of the $5.9 \mathrm{GHz}$ Dedicated 
Short Range Communication (DSRC) Frequency Band, IEEE J. Sel. Areas in Commun., Vol. 25, No. 8, Oct. 2007, 1501-1516.

R. Wang \& D. Cox (2002). Channel modeling for ad hoc mobile wireless networks, in Proc. IEEE Veh. Technol. Conf., pp. 21-25, May 2002.

D. J. Young \& N. C. Beaulieu (2000). The generation of correlated Rayleigh random variates by inverse discrete Fourier transform, IEEE Trans. Commun., Vol. 48, No. 7, Jul. 2000, 1114-1127.

C. S. Patel, S.L. Stuber \& T.G. Pratt (2003). Simulation of Rayleigh faded mobile-to-mobile communication channels, IEEE Veh. Technol. Conf., pp. 163-167, Oct. 2003.

S. Gowrishankar, T. G. Basavaraju and S. K. Sarkar (2007), Effect of Random Mobility Models Pattern in Mobile Ad hoc Networks, Int. J. Computer Science and Network, Vol. 7, No. 6, Jun. 2007, 160-164.

J. Yoon, B. Noble (2006), A General Framework to Construct Stationary Mobility Models for the Simulation of Mobile Networks, IEEE Trans. Mobile Computing, Vol. 5, No. 7, Jul. 2006, 1-12.

K. Pawlikowski, H. D. J. Jeong, and J. S. R. Lee (2002), On credibility of simulation studies of telecommunication networks, IEEE Commun. Mag., Vol. 40, No. 1, Jan. 2002, 132-139.

D. G. Michelson, J. Chuang (2006), Requirements for Standard Radiowave Progpagation Models for Vehicular Environments, in IEEE 63rd Vehicular Technology Conference, Vol. 6, pp. 2777-2781, Sep. 2006.

Network Simulator - ns-2, http://www.isi.edu/nsnam/ns/.

OPNET, http://www.opnet.com.

A. Jardosh, E. M. Belding-Royer, K. C. Almeroth, and S. Suri (2006), Towards realistic mobility models for mobile ad hoc networks, in Proceedings of ACM MobiCom, San Diego, CA, pp. 217íc229, September 2006.

A. P. Jardosh, E. M. Belding-Royer, K. C. Almeroth, and S. Suri (2005), Real-world environment models for mobile network evaluation, IEEE J. Sel. Areas in Commun., Vol. 23, No. 3, Mar. 2005, 622-632.

A. Mahajan, N. Potnis, K. Gopalan and A. Wang (2007), Modeling Vanet deployment in urban settings, in International Workshop on Modeling Analysis and Simulation of Wireless and Mobile Systems, Crete Island, Greece, pp. 151-158, Oct. 2007.

I. Stepanoy, K. Rothermel (2008), On the impact of a more realistic physical layer on MANET simulations results, Ad Hoc Networks, Vol. 6, No. 1, Jun. 2008, 61-78.

G. D. Durgin (2003). Space-Time Wireless Channels, 1st ed.Pearson Education, Inc, 2003. 


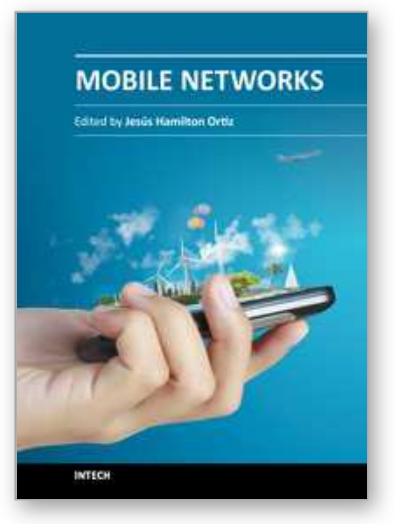

\author{
Mobile Networks \\ Edited by Dr. Jesús Ortiz
}

ISBN 978-953-51-0593-0

Hard cover, 192 pages

Publisher InTech

Published online 09, May, 2012

Published in print edition May, 2012

The growth in the use of mobile networks has come mainly with the third generation systems and voice traffic. With the current third generation and the arrival of the $4 \mathrm{G}$, the number of mobile users in the world will exceed the number of landlines users. Audio and video streaming have had a significant increase, parallel to the requirements of bandwidth and quality of service demanded by those applications. Mobile networks require that the applications and protocols that have worked successfully in fixed networks can be used with the same level of quality in mobile scenarios. Until the third generation of mobile networks, the need to ensure reliable handovers was still an important issue. On the eve of a new generation of access networks (4G) and increased connectivity between networks of different characteristics commonly called hybrid (satellite, ad-hoc, sensors, wired, WIMAX, LAN, etc.), it is necessary to transfer mechanisms of mobility to future generations of networks. In order to achieve this, it is essential to carry out a comprehensive evaluation of the performance of current protocols and the diverse topologies to suit the new mobility conditions.

\title{
How to reference
}

In order to correctly reference this scholarly work, feel free to copy and paste the following:

Yuhao Wang and Xing Xing (2012). Sum-of-Sinusoids-Based Fading Channel Models with Rician K-Factor and Vehicle Speed Ratio in Vehicular Ad Hoc Networks, Mobile Networks, Dr. Jesús Ortiz (Ed.), ISBN: 978-953-510593-0, InTech, Available from: http://www.intechopen.com/books/mobile-networks/sum-of-sinusoids-basedfading-channel-models-with-rician-k-factor-and-vehicle-speed-ratio-in-vehicul

\section{INTECH}

open science | open minds

\section{InTech Europe}

University Campus STeP Ri

Slavka Krautzeka 83/A

51000 Rijeka, Croatia

Phone: +385 (51) 770447

Fax: +385 (51) 686166

www.intechopen.com

\section{InTech China}

Unit 405, Office Block, Hotel Equatorial Shanghai

No.65, Yan An Road (West), Shanghai, 200040, China

中国上海市延安西路65号上海国际贵都大饭店办公楼405单元

Phone: +86-21-62489820

Fax: +86-21-62489821 
(C) 2012 The Author(s). Licensee IntechOpen. This is an open access article distributed under the terms of the Creative Commons Attribution 3.0 License, which permits unrestricted use, distribution, and reproduction in any medium, provided the original work is properly cited. 